\title{
Muerte súbita del lactante: ¿Por qué protege la posición supina?
}

Why the prone position is a risk factor for sudden infant death syndrome?

Jeafrey H, Megevand A, Page M, et al. Pediatrics. Vol. 104 No. 2 August 1999: 263-269

\section{Objetivo}

Intentar explicar el aumento del riesgo de muerte súbita infantil asociado a la posición prona al dormir. Se evaluaron las diferencias en la respuesta de tragar o despertarse mediada por el reflejo de los quimioreceptores laríngeos y la respuesta respiratoria luego de la infusión de agua en posición supina y prona.

\section{Lugar}

Departamento de medicina neonatal del Royal Prince Alfred Hospital de Camperdown y Departamento de Obstetricia y Ginecología de la Universidad de Sydney (Australia).

\section{Pacientes}

Diez infantes de término de tres a cinco días de vida con peso y estatura adecuados para su edad gestacional, nacidos de parto natural de madres no fumadoras que no hubieran tenido complicaciones durante el embarazo y/o recibido analgésicos durante el parto y que no hubieran sido tratados por hiperbilirrubinemia.

\section{Métodos}

Se administraron $0.4 \mathrm{ml}$ de agua esteril en la faringe del neonato durante su sueño cada tres minutos. Luego de ocho infusiones se cambiaba al lactante de posición (de prono a supino o viceversa, según la asignación aleatoria de inicio) y se repetía la operación. El experimento terminaba si el niño se despertaba o cuando se llegaba a las ocho infusiones.

Se controlaba al infante durante los diez segundos posteriores a la infusión registrándose las respuestas del niño (tragar, despertarse, reflejo espiratorio, cambio en la frecuencia respiratoria).

Durante la realización del procedimiento se controlaba a los niños con polisomnografía, parámetros cardiorespiratorios (ej. flujo de aire nasal, movimientos de la caja torácica, frecuencia cardíaca, saturación de oxígeno); así como el tragado y la actividad esofágica a través de un catéter.

\section{Resultados}

De las 164 infusiones; el 95\% evocó una respuesta protectora de tragado y el $54 \%$, de despertar. Hubo diferencias significativas en la respuesta deglutoria (21,3 vs 32 degluciones por minuto) y en la disminución de la frecuencia respiratoria $(-9,6$ vs -2.9 rep. por minuto) entre la posición prona y la supina. No hubo diferencias significativas en el despertar.

\section{Conclusiones}

La protección de la vîa aérea está comprometida en la posición prona, aún en lactantes sanos de término. Esto se debe a una menor respuesta deglutoria en ausencia de un aumento del despertar; lo que podría redundar en un aumento del riesgo de muerte súbita en esta posición.

Fuente de financiamiento: Fundación del Sur de Australia para la Investigación del Síndrome de Muerte Súbita Infantil. Subvención del Consejo Nacional de Salud e Investigación Médica

\section{COMENTARIO}

Tradicionalmente se define a la muerte súbita del lactante como "la muerte súbita de un niño menor de un año de edad a la cual no se le encuentra explicación luego de una detallada investigación que incluye autopsia, exámen de la escena del fallecimiento y revisión de la historia clinica". Es un diagnóstico sindrómico al que se llega por exclusión y que probablemente tenga distintas etiologías.

Es la primera causa de muerte postneonatal (entre 1 mes y un año) en los países desarrollados, representando un 40 a un $50 \%$ de dicha mortalidad ( 1 a 2 cada 1000 nacidos vivos) con predominio masculino. La máxima incidencia se da entre los dos y los cuatro meses. La gran mayoría ocurre entre la medianoche y las 9.00AM en meses fríos y húmedos.

La frecuencia varía geográficamente: es de 3 a 7 por 1000 recien nacidos vivos en Australia, Nueva Zelanda e Irlanda del Norte; los países occidentales tiene una tasa intermedia (1 a 3/1000); y Hong Kong, Japón y Suecia presentan una tasa baja $(0,05$ a 1 cada 1000).1 En Argentina se registraron durante el año 1994, según la dirección de Estadísticas de Salud del Ministerio de Salud y Acción Social de la Nación, 484 defunciones en niños menores de un año de edad categorizadas como muerte súbita de causa desconocida $(0,72$ por cada mil nacidos vivos).

Diferentes hipótesis se plantearon en cuanto a la causa de la muerte súbita, aún desconocida. La única que sí está demostrada es que la posición prona aumenta considerablemente su incidencia. El objetivo de éste trabajo fue evaluar el rol del reflejo quimiolaríngeo y las respuestas respiratorias en posición prona y supina en lactantes sanos a través de diferencias en el quimioreflejo laringeo, un poten- te causal de apnea y bradicardia que luego de una estimulación podría ser fatal si los mecanismos que protegen la vía aérea para la entrada de líquidos (la deglución, el despertar y los reflejos espiratorios) están deprimidos en el niño. La hipótesis de los autores es que los mecanismos protectores de la vía aérea podrían estar deprimidos en posición prona comparado con la supina.

El estudio demostró que en los lactantes sanos, la frecuencia de deglución y la frecuencia respiratoria estan disminuídas significativamente en la posición prona con respecto a la supina, sin diferencias en el despertar. Si bien el hallazgo es estadísticamente significativo y biológicamente plausible, diez pacientes todavía no constituyen una muestra importante como para establecer decididamente la causa de esta entidad. Por otra parte, dentro de los criterios de exclusión no se establece si había antecedentes de muerte súbita en las familias de los participantes.

Evidentemente, recomendar el cambio de posición al dormir ha demostrado su eficacia mundialmente a través de evidencia proveniente de estudios poblacionales ${ }^{1-2}$. En Noruega, por ejemplo el cambio de posición significó una reducción en la incidencia de muerte súbita del lactante (de 3,5 a 0,2 por cada mil nacidos vivos); y en Hong Kong, donde todos duermen en posición supina, la muerte súbita practicamente no existe. En Argentina no conocemos datos sobre su adopción y/o repercusión. En vista de la actual evidencia epidemiológica, e independientemente de la causa, recomendar que el lactante duerma en decúbito supino disminuye la incidencia del síndrome de muerte súbita del lactante y debe incorporarse fuertemente a la práctica del médico de atención primaria.

\section{Dr. Pedro Silberman}

Instructor Residencia de Medicina Familiar y Comunitaria Bahía Blanca.

\section{Referencias}

1. Dwyer T. Ponsonby A. Sudden infant death syndrome: after the back to sleep campaigne. Br M J. 1996; 313:180-181.

2. Skadberg B, Morild I, Markesrad T. Abandoning prone sleeping: effect on the risk of sudden infant death syndrome. J Pediatr. 1998; 132; 340-343. 